\title{
Feasibility and safety of discontinuation of isolation precaution policy for coronavirus disease 2019 (COVID-19) patients from COVID-19 units to general medical units in Thailand
}

\author{
Anucha Apisarnthanarak MD ${ }^{1}$ (D), David K. Warren MD, MPH² and David J. Weber MD, MPH ${ }^{3}$ \\ ${ }^{1}$ Division of Infectious Diseases, Thammasat University Hospital, Pathum Thani, Thailand, ${ }^{2}$ Division of Infectious Diseases, Washington University School of \\ Medicine, St Louis, Missouri, United States and ${ }^{3}$ Gillings School of Global Public Health, Chapel Hill, North Carolina, United States
}

To the Editor-Discontinuation of airborne isolation precautions for coronavirus disease 2019 (COVID-19) patients in COVID-19 units is of great importance because the burden of COVID-19 cases that need acute care has significantly increased in Thailand since July 4, 2021. The abrupt increase in COVID-19 cases that have required acute care has exceeded the capacity of care in the COVID-19 units in most Thai hospitals. The principle of discontinuation of isolation precautions for COVID-19 patients is based on the current understanding of the duration of infectivity of severe acute respiratory coronavirus virus 2 (SARS-CoV-2). ${ }^{1}$ In reality, not many Thai hospitals have established discontinuation of isolation precaution policies. Healthcare personal (HCP) have faced problems with unnecessarily prolonged isolation precautions for COVID-19 patients. This delay is frustrating for patients, consumes personal protective equipment, and compromises the safety of patients receiving care in COVID-19 units, due to the delays in laboratory collection and reporting, and critical care management. ${ }^{2}$ These limitations have to be taken into consideration, along with the safety of HCP who may acquire SARS-CoV-2 from patients if they are prematurely released from isolation precautions. We report here the feasibility and safety of a discontinuation of isolation precautions policy implemented in a tertiary-care hospital in Thailand.

Thammasat University Hospital, Pratum Thani, Thailand, has an 18-bed COVID-19 ICU that cares for severely ill COVID-19 patients, a 20-bed unit that cares for moderately ill COVID-19 patients, and a 30-bed COVID-19 unit that cares for mildly ill COVID-19 patients. On April 1, 2021, a discontinuation policy for COVID-19 isolation precaution was initiated, based on existing scientific evidence., ${ }^{3,4}$ This policy allows (1) the discontinuation of isolation precautions for mildly-to-moderately ill COVID-19 patients at day 14 from symptom onset or from the first detection of RT-PCR and (2) the discontinuation of isolation precautions for severely ill and severely immunocompromised COVID-19 patients at day 21 from symptom onset or from the first detection of SARSCoV-2 by RT-PCR. This is a time-based strategy. Under this policy, discontinuation of isolation precautions for COVID-19 patients requires infectious diseases consultant's approval and does not require test-based clearance (ie, a negative SARS-CoV-2 RT-PCR). However, a test-based clearance scheme could be used in special cases in which early or delayed discontinuation of

Author for correspondence: Anucha Apisarnthanarak, E-mail: anapisarn@yahoo.com Cite this article: Apisarnthanarak A, Warren DK, and Weber DJ. (2022). Feasibility and safety of discontinuation of isolation precaution policy for coronavirus disease 2019 (COVID-19) patients from COVID-19 units to general medical units in Thailand. Infection Control \& Hospital Epidemiology, 43: 1732-1733, https://doi.org/10.1017/ ice. 2021.335
Table 1. Healthcare Personal and Patients' Demographics and Characteristics

\begin{tabular}{|c|c|}
\hline Variable & No. $(\%)^{a}$ \\
\hline \multicolumn{2}{|l|}{ Healthcare personal $(n=69)$} \\
\hline Age, median y (range) & $32(22-49)$ \\
\hline Sex, female & $59(85)$ \\
\hline \multicolumn{2}{|l|}{ Underlying diseases } \\
\hline None & $59(85.6)$ \\
\hline Hypertension & $2(2.8)$ \\
\hline Diabetes & $6(8.6)$ \\
\hline Other ${ }^{b}$ & $3(4.3)$ \\
\hline BMI, median kg/m² (range) & $24(20-28)$ \\
\hline $\begin{array}{l}\text { Duration of care for COVID-19 patients, median d } \\
\text { (range) }\end{array}$ & $6(2-12)$ \\
\hline Development of COVID-19 symptoms & $0(0)$ \\
\hline \multicolumn{2}{|l|}{ Patients $(n=25)$} \\
\hline Age, median y (range) & $59(24-79)$ \\
\hline Sex, female & $15(60)$ \\
\hline \multicolumn{2}{|l|}{ Underlying diseases } \\
\hline Diabetes & $15(60)$ \\
\hline Hypertension & $10(40)$ \\
\hline Pulmonary diseases & $9(24)$ \\
\hline Gastrointestinal diseases & $6(25)$ \\
\hline Others $^{c}$ & $5(20.8)$ \\
\hline Immunocompromised status $^{d}$ & $2(8)$ \\
\hline BMI (median, range; kg/m²) & $28(2-32)$ \\
\hline \multicolumn{2}{|l|}{ Severity strata } \\
\hline Mild COVID-19 & $0(0)$ \\
\hline Moderate COVID-19 & $10(40)$ \\
\hline Severe COVID-19 & $15(60)$ \\
\hline $\begin{array}{l}\text { Test-based clearance for discontinuation of isolation } \\
\text { precaution }\end{array}$ & $0(0)$ \\
\hline \multicolumn{2}{|l|}{ Place of referral } \\
\hline Intensive care unit & $15(60)$ \\
\hline General medical units & $10(40)$ \\
\hline Died & $2(8)$ \\
\hline
\end{tabular}

Note. BMI, body mass index.

${ }^{a}$ No. (\%) unless indicated otherwise.

bystemic lupus erythromatosus, rheumatic diseases, migraine.

'Stroke, cancers, human immunodeficiency syndromes.

${ }^{\mathrm{d} C a n c e r s}$ on several immunosuppressants. 
isolation precaution for immunocompromised patient is being considered. After discontinuation of isolation precautions, HCP use standard precautions in the general medical units when caring for COVID-19 patients. HCP are prospectively followed for the development of COVID-19 symptoms and an RT-PCR for SARS-CoV-2 is performed if COVID-19 symptoms develop. Transmission-based precautions (eg, contact precautions for multidrug-resistant pathogens) are also used, if indicated.

Prior to the initiation of the discontinuation of isolation precaution policy, we performed a workshop to share the evidence base for the policy and the elements of the policy with nursing staff throughout the general medical units. Then we collected data including HCP and patient demographics, underlying disease data, classification of COVID-19 severity, ${ }^{5}$ immunocompromised status among COVID-19 patients, development of COVID-19 symptoms in HCP over 2 weeks after the end of patient care, evidence of SARS-CoV-2 RT-PCR among HCP (if performed), as well as complaints and barriers reported by HCP after the policy was implemented.

From April 1, 2021, to July 4, 2021, 25 COVID-19 patients were admitted to the general medical units and $69 \mathrm{HCP}$ were involved in the care of COVID-19 patients. The median age of these HCP was 32 years (range, $22-49$ years), and 59 (86\%) of these $69 \mathrm{HCP}$ had no underlying disease(s). For these 25 COVID-19 patients, the median age was 59 years (range, $24-79$ ); 15 patients $(60 \%)$ were female; their median body mass index was $28 \mathrm{~kg} / \mathrm{m}^{2}$ (range, 24-32), and 23 (92\%) had underlying disease(s). Also, 15 patients (60\%) received ICU care: 13 of these were classified as severely ill and 2 were immunocompromised. Furthermore, 10 patients (40\%) were considered moderately ill and received care in a general medical unit. None of the patients had test-based clearance prior to discontinuation of isolation precaution. HCP and patients demographics are summarized in Table 1. During the follow-up period, none of the HCP reported COVID-19 symptoms or had a SARS-CoV-2 RT-PCR performed. Finally, 2 severely ill COVID-19 patients (8\%) died from sepsis with multiorgan failure in the ICU. HCP reported no significant complaints or limitations in the care of COVID-19 patients in the ICU and in general medical units throughout the hospital during this period.

In this study, a time-based policy for discontinuation of COVID19 isolation precautions for infected patients in COVID-19 ICUs and general medical units was feasible and safe for HCP who cared for these patients. Such policies have become important because increasing COVID-19 cases that require acute care may exceed the maximum hospital capacity to care for these patients in COVID-19 units. In addition, once the policy was established, no significant barriers arose and no resistance to this policy by HCP occurred. Notably, although patients in our cohort were obese, had underlying diseases, and were mostly classified as severely ill or immunocompromised, none of our cases had repeat SARSCoV-2 RT-PCR tests to help guide discontinuation of isolation precautions. Our data support the recommendation by the US Centers for Disease Control and Prevention to avoid test-based clearance, given that persistently PCR-positive patients are not contagious. ${ }^{1-4}$ A limitation of our study is that we cannot exclude the possibility of asymptomatic infections in our HCP because routine SARS-CoV-2 testing was not performed. However, because we have no evidence of an outbreak of COVID-19 among our HCP, we believe that the time-based strategy of discontinuation of COVID-19 precautions was both effective and safe. Additional studies with larger sample sizes, from various settings, and with various COVID-19 strains are needed to reassure the feasibility and safety of such policies in acute-care facilities across all continents.

Acknowledgments.

Financial support. No financial support was provided relevant to this article.

Conflicts of interest. All authors report no conflicts of interest relevant to this article.

\section{References}

1. Rhee C, Kanjilal S, Baker M, Klompas M. Duration of severe acute respiratory syndrome coronavirus 2 (SARS-CoV-2) infectivity: when is it safe to discontinue isolation? Clin Infect Dis 2021;72:1467-1474.

2. Apisarnthanarak A, Chaononghin S, Katawethiwong P, Warren DK. High mortality in COVID-19-suspected unit: lessons learned for patient safety. Infect Control Hosp Epidemiol 2021. doi: 10.1017/ice.2021.91.

3. Clinical management of COVID-19 (interim guidance). World Health Organization website. https://www.who.int/publications/i/item/WHO2019-nCoV-clinical-2021-1. Published May 27, 2020. Accessed July 19, 2021.

4. Discontinuation of transmission-based precautions and disposition of patients with COVID-19 in healthcare settings (interim guidance). Centers for Disease Control and Prevention website. https://www.cdc.gov/coronavirus/2019-ncov/ hcp/disposition-hospitalized-patients.html. Updated August 10, 2020. Accessed June 4, 2021.

5. Clinical spectrum of SARS-CoV2-2 infection. National Institutes of Health website. https://www.covid19treatmentguidelines.nih.gov/overview/clinicalspectrum/. Accessed June 4, 2021.

\title{
A look into the relationship between outdated coronavirus disease 2019 (COVID-19) treatment protocols and the overwhelming rise of mucormycosis in COVID-19 patients in India
}

\author{
Saad Ahmed Jamal MBBS (1) and Aashish Rayapati MBBS \\ Independent Scholar
}

\footnotetext{
Author for correspondence: Saad Ahmed Jamal, E-mail: saad.gx@gmail.com Cite this article: Jamal SA and Rayapati A. (2022). A look into the relationship between outdated coronavirus disease 2019 (COVID-19) treatment protocols and the overwhelming rise of mucormycosis in COVID-19 patients in India. Infection Control \& Hospital Epidemiology, 43: 1733-1735, https://doi.org/10.1017/ice.2021.385
}

To the Editor - India, the second most-affected country by coronavirus disease 2019 (COVID-19), recorded one of the worst second waves across the world, with $>400,000$ daily cases reported in early 